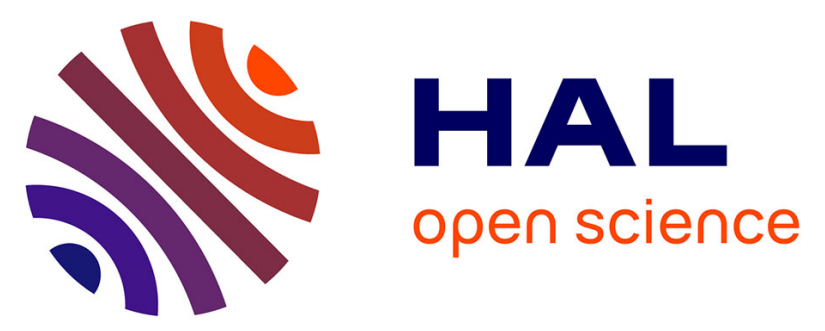

\title{
Interfacial adhesion between the iron fillers and the silicone matrix in magneto-rheological elastomers at high deformations
}

Tobias Pössinger, Christian Bolzmacher, Laurence Bodelot, Nicolas

Triantafyllidis

\section{To cite this version:}

Tobias Pössinger, Christian Bolzmacher, Laurence Bodelot, Nicolas Triantafyllidis. Interfacial adhesion between the iron fillers and the silicone matrix in magneto-rheological elastomers at high deformations. SPIE Microtechnologies Conference,, Apr 2013, Grenoble, France. pp.87631Y-11, 10.1117/12.2016532 . hal-00870133v2

\section{HAL Id: hal-00870133 \\ https://hal.science/hal-00870133v2}

Submitted on 8 Oct 2013

HAL is a multi-disciplinary open access archive for the deposit and dissemination of scientific research documents, whether they are published or not. The documents may come from teaching and research institutions in France or abroad, or from public or private research centers.
L'archive ouverte pluridisciplinaire $\mathbf{H A L}$, est destinée au dépôt et à la diffusion de documents scientifiques de niveau recherche, publiés ou non, émanant des établissements d'enseignement et de recherche français ou étrangers, des laboratoires publics ou privés. 


\title{
Interfacial adhesion between the iron fillers and the silicone matrix in magneto-rheological elastomers at high deformations
}

\author{
Tobias Pössinger* ${ }^{\mathrm{a}}$, Christian Bolzmacher ${ }^{\mathrm{a}}$, Laurence Bodelot ${ }^{\mathrm{b}}$, Nicolas Triantafyllidis ${ }^{\mathrm{b}}$ \\ ${ }^{a}$ CEA, LIST, Sensorial and Ambient Interfaces Laboratory, 91191 Gif-sur-Yvette CEDEX, France; \\ 'École Polytechnique, Laboratoire de Mécanique des Solides (LMS), Route de Saclay, F-91128 \\ Palaiseau, France
}

\begin{abstract}
This work investigates the interfacial adhesion between the iron fillers and the silicone matrix in magneto-rheological elastomers at high deformations. Carbonyl iron powder, composed of mechanically soft spherical particles with a median size of $3.5 \mu \mathrm{m}$ and a volume concentration of 3.5\%, was mixed in a soft silicone matrix (Shore 00-20); the compound was then degassed and cured under temperature. The presence of a homogeneous magnetic field of $0.3 \mathrm{~T}$ during the curing process allowed the formation of particle chains. Tensile tests of these samples under scanning electron microscope showed interfacial slipping and debonding between the two phases. To improve interfacial adhesion, a silane primer was applied to the iron particles, following two different procedures, before the mixing and crosslinking process, thus giving two additional types of samples. In tensile testing lengthwise to the particle alignment, with engineering strains up to $150 \%$, the structural responses of the different types of samples were compared. An enhanced adhesion of the iron fillers to the silicone matrix resulting in a reinforced matrix and increased tensile strength during the first loading path could be observed. Furthermore, scanning electron microscope images show that a more elaborated particle-matrix interface was obtained with the primer additive.
\end{abstract}

Keywords: magneto-rheological elastomer, silicone elastomer, carbonyl iron powder, silane primer, interfacial adhesion, high deformation

\section{INTRODUCTION}

Materials whose rheological properties can be varied by the application of magnetic fields belong to the specific class of smart materials because they can respond rapidly and reversibly to changes in their environment. A branch of this kind of materials, called magneto-rheological elastomers (MRE), are typically composed of micron-sized magnetic particles dispersed in an elastomeric matrix. When a magnetic field is applied to the polymer composite during crosslinking, particle chain structures form and remain locked in place upon final curing, thus imparting anisotropic properties to viscoelastic materials $[1,2]$.

Not only do magneto-rheological elastomers alter their viscoelastic properties in response to an external magnetic field but they can also undergo very high deformation states. While the former effect is mainly exploited in variable and controllable stiffness devices [1-3], the latter may be of interest in haptic devices such as a tactile interface [4]. Indeed, a large out-of-plane deformation can be introduced when the material is chosen appropriately. Using a coil or a permanent magnet matrix placed underneath a soft MRE surface, patterns can be displayed on the surface as already achieved with magneto-rheological fluids [5].

It is well known that the distribution, size and shape of the filler particles, as well as the state of the interface between the particles and matrix, affect the macroscopic behavior of composite materials [6,7]. Indeed, filled elastomers responses are typically exhibiting the Payne effect at small deformations [8] and the Mullins effect at high deformations [9, 10]. Moreover, at a critical stress level, debonding acts as a distinct failure phenomenon in an elastomer containing rigid inclusions, due to stress concentrations at the weak particle-matrix interface [11]. Therefore the phenomenon of debonding, bound and entrapped rubber appears as a key property for the behavior of filler-rubber compounds [12, 13] and has actually been observed in MREs [14]. 
Besides, the common use of silicone oil as a diluting agent to decrease the modulus of silicone elastomers tends to enhance by facilitating frictional sliding at the interfaces between the matrix and the particles [15-18].

In order to modify the particle-matrix interactions and more precisely to improve the interfacial adhesion, different kinds of silane coupling agents and compatibilizer were used in MRE [19, 20]. Silane primers typically consist of two different reactive groups, one compatible to the substrate and the other to the elastomer and serve as adhesion promoters between two nonbonding surfaces. Some studies showed the improved bond strength between many primed substrates and addition-cure silicon elastomer [21, 22].

In the perspective of developing a tactile MRE surface, this work investigates, as a first step, the interfacial adhesion between the iron fillers and the silicone matrix at high deformations. Therefore different MRE samples were prepared by modifying the surface of carbonyl iron particles (CIP) through silane primer treatment according to two procedures, prior to compounding. These samples are then compared to a sample without particle treatment both under SEM and during mechanical testing.

In the following, we detail in part 2 the experimental procedures for sample preparation, SEM observation and mechanical testing before presenting and discussing our results in part 3.

\section{EXPERIMENTAL}

\subsection{Materials}

The very soft and stretchable silicone elastomer Ecoflex 0020, purchased from Smooth-On, Inc., USA was chosen as the matrix material to facilitate high deformations under reasonable magnetic field for a possible application as a tactile MRE surface. It is cured as a two-part vulcanization addition cure platinum-catalyzed system (RTV-2). The obtained silicone has a Shore hardness of 00-20, an elongation at break of $845 \%$ and a density $\rho_{\text {sil }}$ of about $1.07 \mathrm{~g} / \mathrm{cm}^{3}$.

Mechanically soft, spherical carbonyl iron powder (CIP) with a median particle size of $3.5 \mu \mathrm{m}$ and a density $\rho_{\text {iron }}$ of about $7.5 \mathrm{~g} / \mathrm{cm}^{3}$ from BASF, Germany, was used as the filler material.

To improve adhesion between the silicone elastomer and the iron particles, the clear 1200 OS Primer from Dow Corning, USA, was used. The 5\% non-volatile active content of this silane based primer is purchased diluted in volatile siloxanes.

\subsection{Samples preparation}

A pure silicone elastomer and different MRE samples were prepared modifying, or not, the surface of CIP by silane primer treatment of the particles prior to compounding. The silane primer dilution was applied either by spray-coating the particles or by stirring them in the primer dilution, giving the two procedures mentioned in the introduction. In order to observe the MREs' microstructures clearly under microscope, a low particle volume fraction of 3.5\% was used and samples were cured in the presence of a magnetic field of $0.3 \mathrm{~T}$.

The different types of samples used in this work are summarized in Table 1 and details regarding their fabrication are given in the following paragraphs.

Table 1: Volume fractions and particle pretreatment of the different types of samples prepared for further investigation with scanning electron microscopy and mechanical measurements.

\begin{tabular}{|c|c|c|c|c|}
\hline & pure silicone & untreated MRE & spray-coated MRE & primer stirred MRE \\
\hline silicone elastomer & $100 \%$ & $96.5 \%$ & $96.5 \%$ & $96.5 \%$ \\
\hline $\begin{array}{c}\text { CIP volume } \\
\text { fraction }\end{array}$ & $0 \%$ & $3.5 \%$ & $3.5 \%$ & $3.5 \%$ \\
\hline $\begin{array}{c}\text { silane primer } \\
\text { treatment of CIP }\end{array}$ & none & none & $\begin{array}{c}\text { particles sprayed by } \\
\text { dilution }\end{array}$ & $\begin{array}{c}\text { particles stirred in } \\
\text { dilution }\end{array}$ \\
\hline
\end{tabular}


Pure silicone: A pure silicone material sample without filler was prepared. The polymer (Part A) and the crosslinker (Part B) were dispensed in a 1:1 weight ratio and thoroughly mixed for 3 minutes by a mechanical stirring machine at $400 \mathrm{rpm}$. Then, the mixed material was degassed at 1 mbar for 8 minutes to eliminate any entrapped air and poured by hand in a copper-Teflon mold presented in Figure 1 . The curing was accelerated by heating the mold at $100{ }^{\circ} \mathrm{C}$ via a thermoelectric power generation system described in Figure 1. The samples were finally demolded having a volume of $24 \times 24 \times 4 \mathrm{~mm}^{3}$ (see also Figure 3).
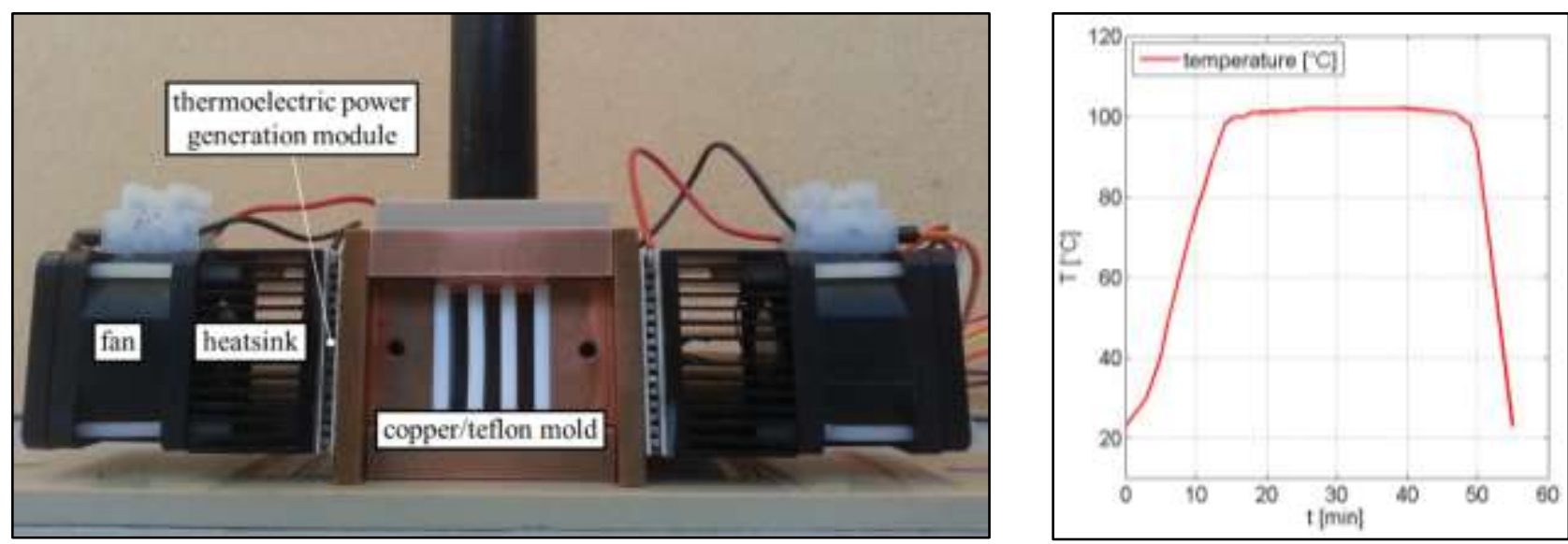

Figure 1: Thermoelectric power generation system (left) and heat treatment (right).

Untreated MRE: A series of MRE samples without silane particle treatment was prepared by adding $40 \%$ in weight of silicone (Part A), 40\% in weight of crosslinker (Part B) and 20\% in weight of iron particles. This weight fraction of $20 \%$ corresponds to a volume fraction of about $3.5 \%$ of carbonyl iron particles. The material was mixed, degassed and cured as the pure silicone samples, but under a constant magnetic field of about 0.3T. The magnetic field was realized via a coil/permanent magnet device presented in Figure 2 in order to align the particles in chain-like structures. A simulation via FEMM software shows that the field is homogeneous in the zone where the samples are cured (see Figure 2).
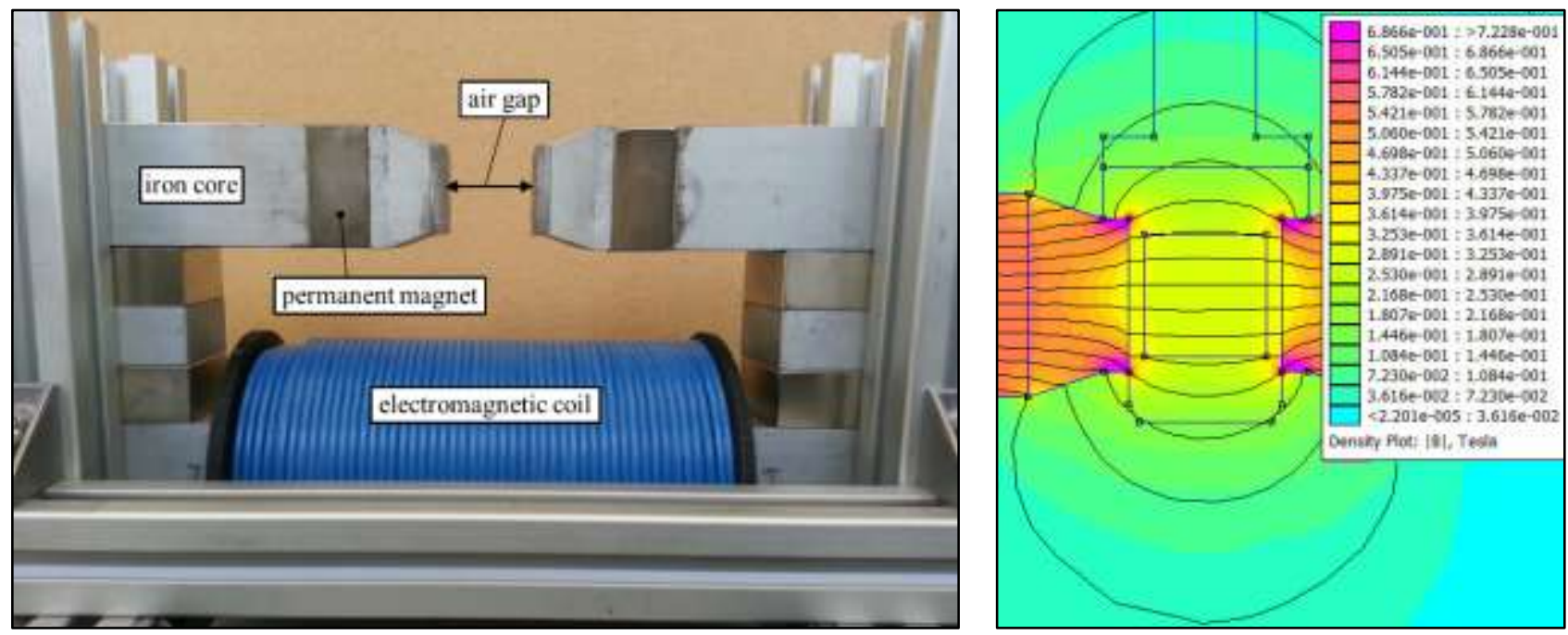

Figure 2: Coil/permanent magnet device (left) and FEMM-simulation showing the homogeneity of the magnetic flux inside the air gap (right). 
Spray-coated MRE: A series of MRE samples was prepared by dispersing the particles in a plastic container and spraying the primer through an air brush onto the particles while shaking the container. After 45 min of air drying of the primer treated particles, $40 \%$ in weight of silicone (Part A), $40 \%$ in weight of crosslinker (Part B) and $20 \%$ in weight of pretreated carbonyl iron particles were mixed, degassed and cured as the untreated MRE samples.

Primer stirred MRE: Another series of MRE samples was prepared by directly stirring the iron particles in the silane primer dilution. After the stirring process, the particle-primer-mix was widely dispersed on a Teflon plate, rinsed out with Ethanol and air dried for 45 minutes. Then, $40 \%$ in weight of silicone (Part A), 40\% in weight of crosslinker (Part B) and $20 \%$ in weight of pretreated iron particles were mixed, degassed and cured as the untreated MRE samples.

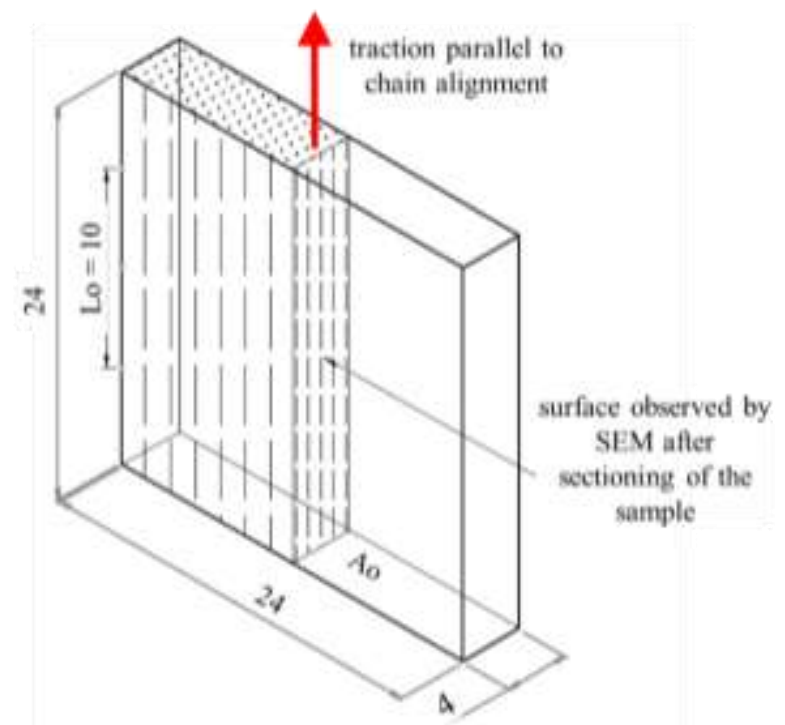

Figure 3: Sample geometry (dimensions in $\mathrm{mm}$ ) for SEM observation and mechanical measurements.

\subsection{Scanning electron microscopy}

To evaluate the effect of primer application and the subsequent interfacial adhesion between the iron fillers and the silicone matrix both the untreated and primer treated particles as well as the 3 types of MRE samples described in 2.2 were observed by using an environmental scanning electron microscope (ESEM, model MEB Quanta 600 FEG). The observation of untreated and primer treated particles was realized under high-vacuum mode. A beam voltage of $5 \mathrm{kV}$ and the Everhart-Thornley detector were used. In order to limit charge phenomena during the imaging of low conductivity material, the observation of the MRE samples was realized under low-vacuum mode with a chamber pressure of $130 \mathrm{~Pa}$ and environmental water vapor. A beam voltage of $10 \mathrm{kV}$ and a back-scattered electron detector were used. For the observation of the particle chains the samples were cut in half parallel to chain orientation using a scalpel. The surface observed in the SEM is highlighted in Figure 3. An in-situ traction device allowed pulling on the MRE samples parallel to the chain orientation up to $140 \%$ strain so as to observe their microstructure at high deformation states.

\subsection{Mechanical measurements}

For the comparison of the structural responses of the different MRE samples and the pure silicone elastomer, traction tests were performed at room temperature (about $23{ }^{\circ} \mathrm{C}$ ). The samples were maintained by mechanical grips separated by an initial distance $\mathrm{L}_{0}$ of about $10 \mathrm{~mm}$. The elongation of the sample was measured by the displacement of the motors to which the grips were attached with an accuracy of less than $50 \mu \mathrm{m}$. The tensile force was measured by a $10 \mathrm{~N}$ universal load cell (Althen ALF328) with an accuracy of less than $50 \mathrm{mN}$. The nominal stress was calculated as the ratio of the axial force to the undeformed cross-sectional area $\mathrm{A}_{0}$ of the samples, that is $24 \times 4 \mathrm{~mm}^{2}$ (see Figure 3 ). The nominal strain was calculated as the ratio of the current elongation to the initial length. The samples were subjected to sinusoidal cyclic loading and unloading from the unstrained state up to a maximum strain of $150 \%$ at a strain rate of $0.02 \mathrm{~s}^{-1}$. 


\section{RESULTS AND DISCUSSION}

\subsection{Macroscopic pre-investigations}

Macroscopic investigations were conducted at the beginning of this study to clearly illustrate the phenomenon of interfacial slipping and debonding of the soft silicone matrix from rigid spherical inclusions (Figure 4). Spheres of magnetic steel, as used in ball bearings, with a diameter of $1.5 \mathrm{~mm}$ were molded in the Ecoflex 0020 silicone elastomer and observed under traction up to $40 \%$. It can be observed, that there is a strong debonding between the spheres and the elastomeric matrix, starting already at small strains and being more pronounced around large particle agglomerates. It is worth mentioning here that this test with large beads was for illustrative purpose only. As a matter of fact, a very important parameter for the adhesion between the filler particle and the rubber is the particle size.
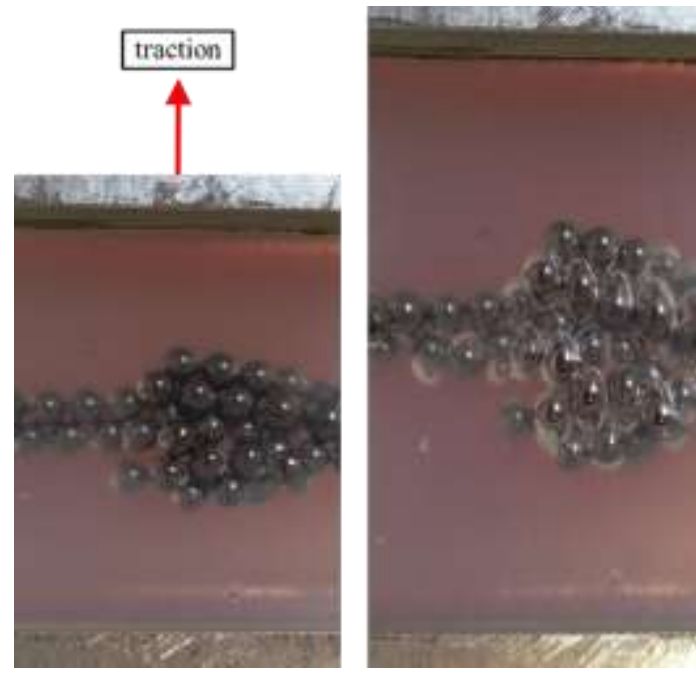

Figure 4: Debonding of the silicone elastomer from $1.5 \mathrm{~mm}$ spheres at traction.

Further investigations focused on the strength of the bond between the silicone matrix material to be employed and an iron plate either untreated or treated with primer. The two pure iron plates (Telar 57) were polished to an average surface finish of about $0.125 \mu \mathrm{m}$ to imitate the surface of the small carbonyl iron particles. The silicone elastomer was then cast between the two plates with an area of $18 \times 18 \mathrm{~mm}^{2}$ at a distance of $3 \mathrm{~mm}$ (Figure 5). During preliminary traction tests, the debonding of an untreated and a primer treated surface (brushing with silane primer and air drying) of the iron plates was studied. In the stress-strain curves from Figure 3 (on the right-hand side), it can be observed that the adhesion of the silicone to the primer treated surface is increased compared to the untreated surface by a factor of 8 . At a strain of about $80 \%$, the silicone elastomer starts to debond from the untreated iron surface by slowly peeling off. In the case of the primer treated iron surface, the silicone elastomer does not start to debond until a strain of about $520 \%$.
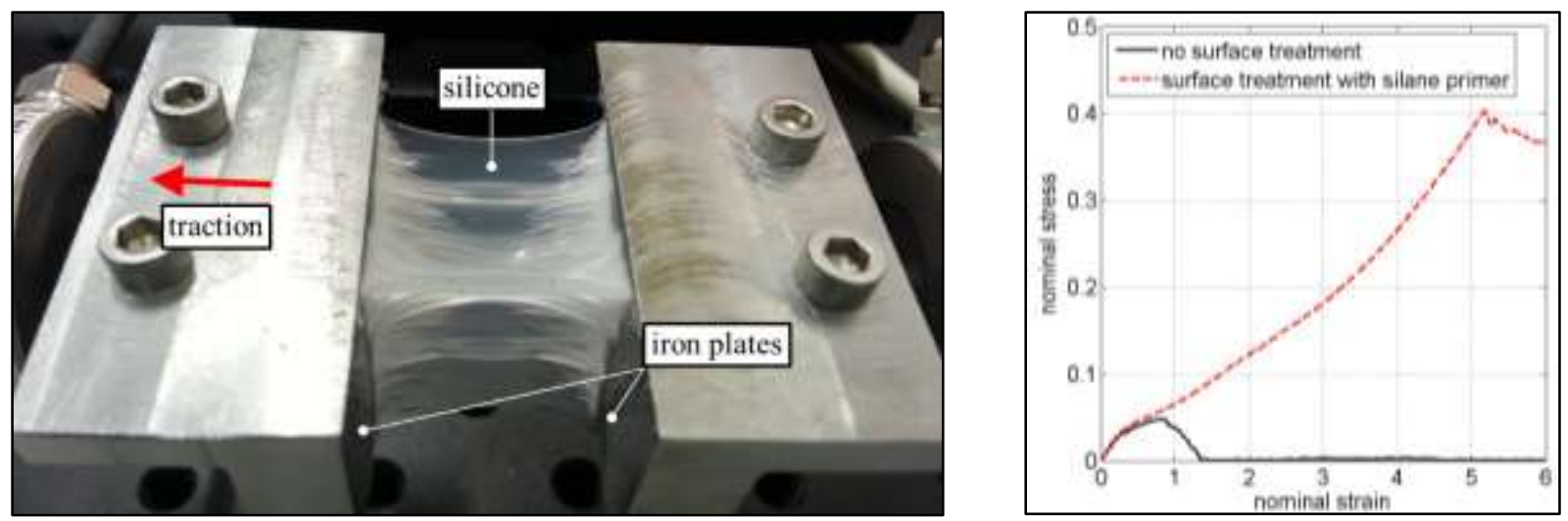

Figure 5: Increased bond strength between a primer treated iron surface and the silicone used in this study. 


\subsection{Scanning electron microscopy (SEM)}

To evaluate the effect of the primer application both the untreated and primer treated particles were observed by scanning electron microscope and are confronted in Figure 6. While the untreated (Figure 6a) and spray-coated (Figure $6 \mathrm{~b})$ particles are loosely assembled, the primer stirred particles are stuck together in irregular agglomerates by the nonvolatile primer content (Figure 6c). Very few spray-coated particles tend to be covered by the primer as shown in Figure 6b. Only a few well covered particles or particle agglomerates can actually be found among the spray-coated particles, they are shown in Figure 7. The majority of the primer stirred particles are covered by the silane primer however rather poorly as shown in Figure 6c.
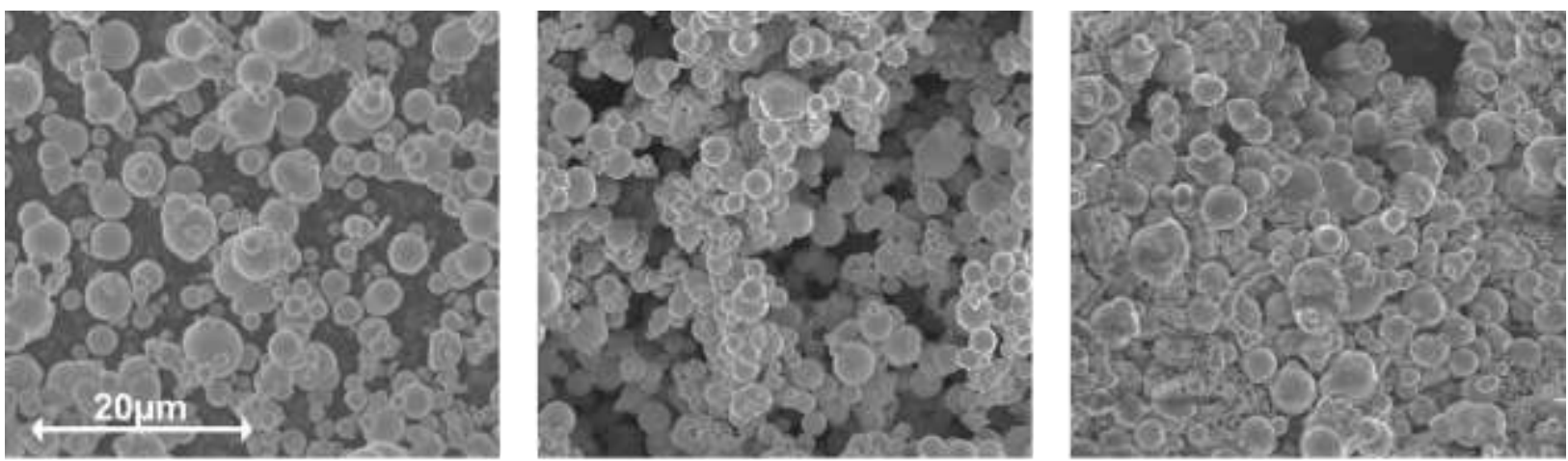

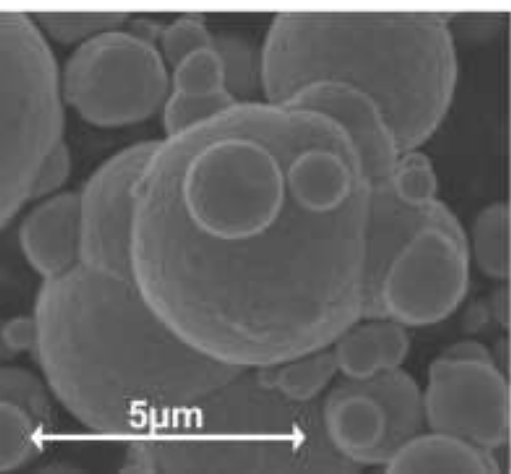

a. untreated particles

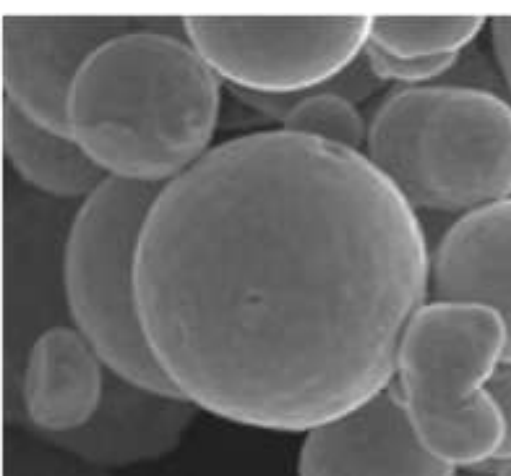

b. spray-coated particles

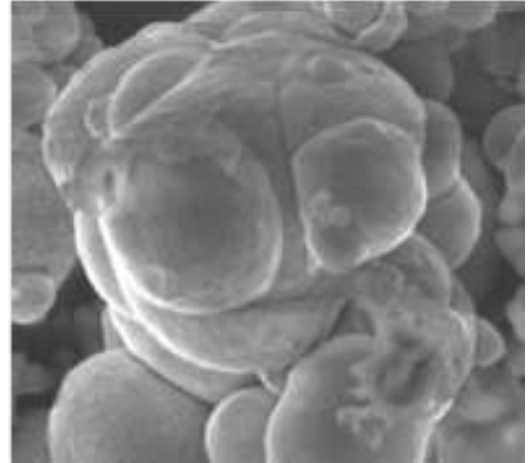

c. primer stirred particles

Figure 6: SEM observation of the silane primer application on the carbonyl iron particles with a median particle size of 3.5 $\mu \mathrm{m}$ at a magnification of 1,500 (top) and 6,000 (down).
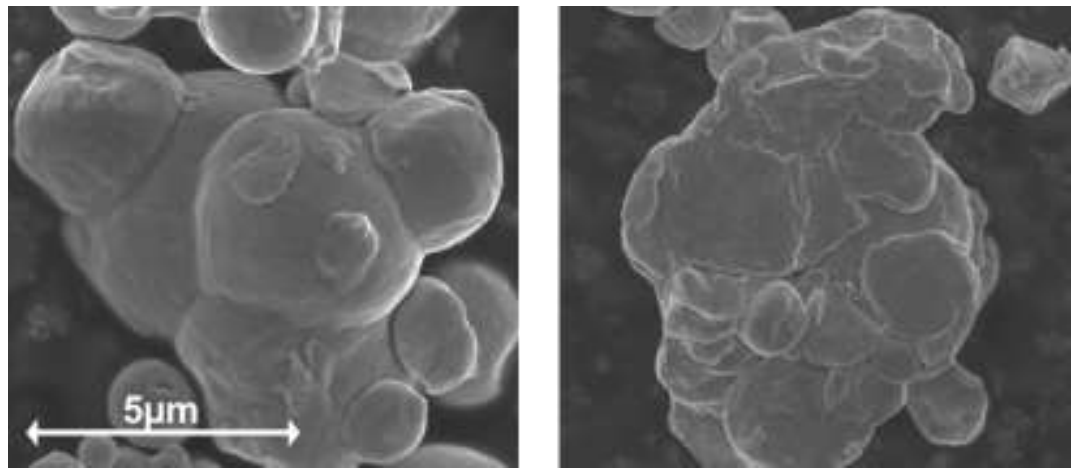

Figure 7: SEM images of the few particles and particle agglomerates well covered by the primer found among the spraycoated particles at a magnification of 6,000 . 
The SEM images of the MREs with untreated (Figure 8a), spray-coated (Figure 8b) and primer stirred (Figure 8c) particles without any in-situ traction show the influence of the primer treatment on chain alignment. The dark silicone in the back highlights the iron particles appearing as white spots. There is no remarkable difference in chain alignment between the untreated and the spray-coated samples. The particles cured under a magnetic field of $0.3 \mathrm{~T}$ are aligned in thin, disconnected chains spaced with small distances. In contrast, particle chains in the primer stirred MRE create thick columns of irregular particle agglomerates with higher spatial distances.

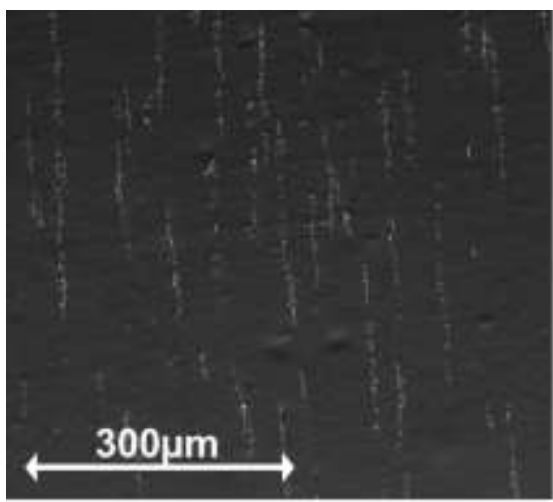

a. untreated MRE

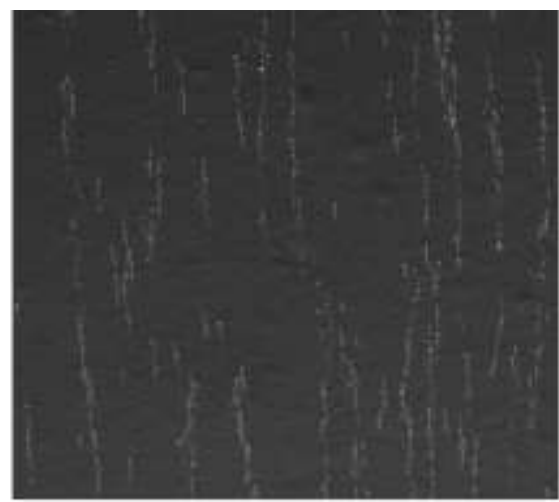

b. spray-coated MRE

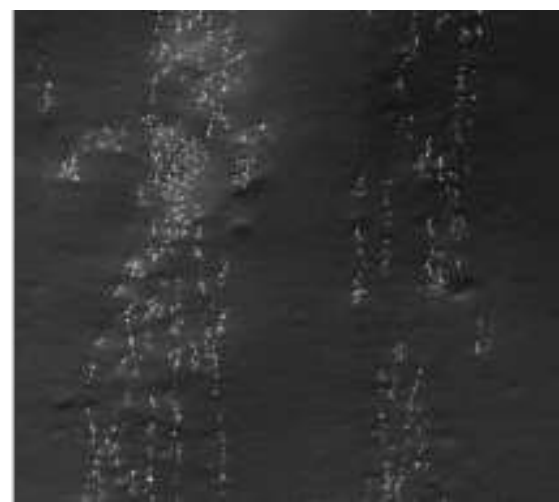

c. primer stirred MRE

Figure 8: Particle chain alignment of the untreated and silane treated MRE samples with a particle volume fraction of 3.5\% at a magnification of 200 .

To study the interfacial adhesion between the iron fillers and the silicone matrix at high deformation states, the different MRE samples were observed under SEM at a nominal strain of $140 \%$ applied with an in-situ traction device, as illustrated in Figure 9. Fracture initiation and nucleation at the surface of the MRE samples as a result of the in-situ traction can be observed; the first cracks actually appear at a strain of 50\%. The initiation of cracks is very likely caused by the initial scalpel cut and the following deterioration of the samples by the electron beam. The subsequent nucleation of cracks at the MRE surface as a result of the in-situ traction allowed a further investigation of the interfacial adhesion between the iron filler and the elastomeric matrix at a local scale. The silicone matrix is detached from the particles for the untreated (Figure 9a) and the spray-coated MREs (Figure 9b) without any remaining residues. However, in the case of the spray-coated MRE, where only very few well bound particles could be observed (Figure 7), we can detect a few particles to which the matrix is still rather well attached (Figure 10). In the case of the primer stirred MRE, bigger particles and particle agglomerates appear more prone to the formation of cracks. Though the majority of the particles show residues of bound rubber at the interfaces. The matrix adheres only in separate filaments to the poorly primer covered surface (Figure 9c). These observations are in agreement to the previous SEM images of the different particle treatments before compounding (Figure 6, Figure 7). 

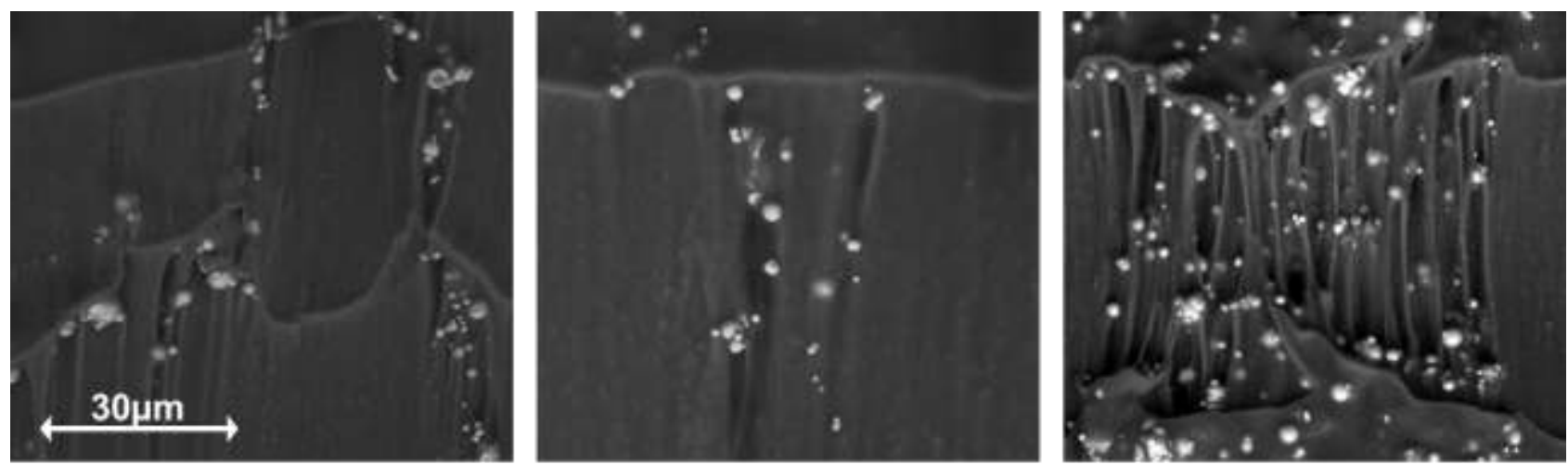

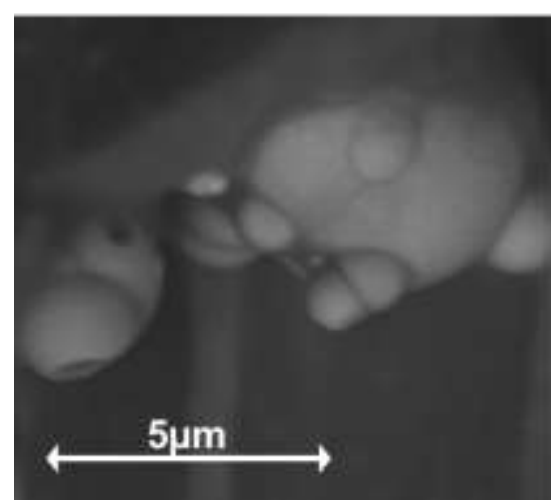

a. untreated MRE

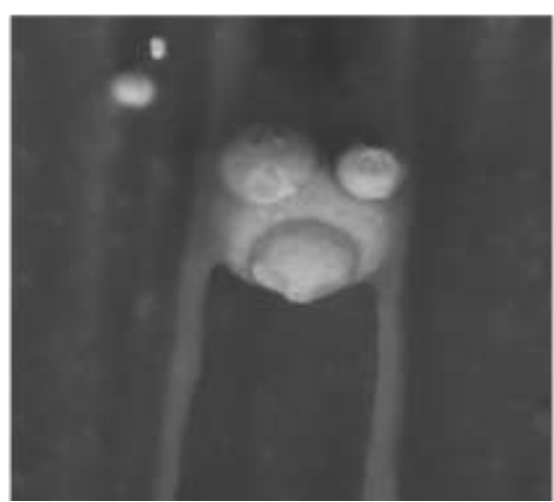

b. spray-coated MRE

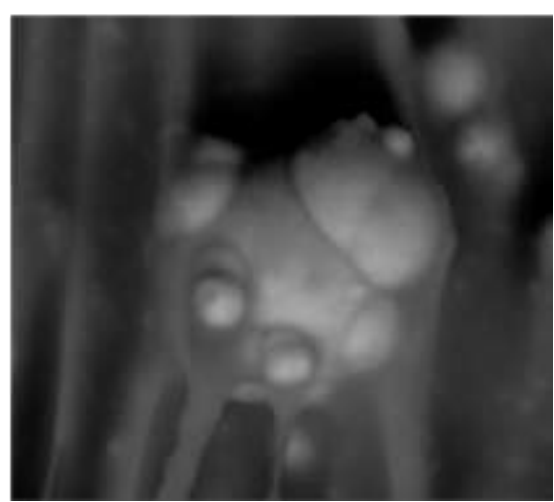

c. primer stirred MRE

Figure 9: Interfacial adhesion between the iron filler and the silicone matrix of the different MRE samples at a strain of $140 \%$ and a magnification of 1,500 (top) and 12,000 (down).
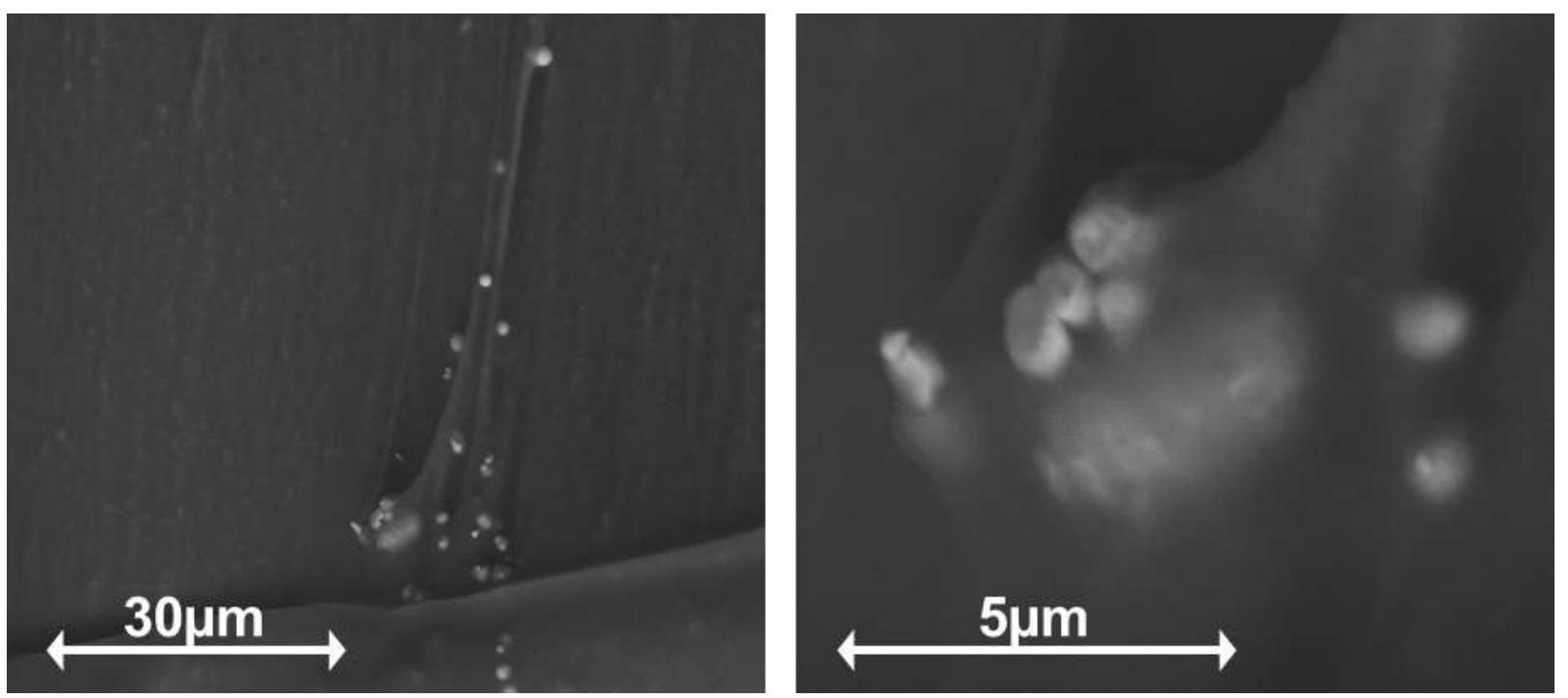

Figure 10: SEM images of the few well bound particles and particle agglomerates found among the spray-coated MRE sample at a magnification of 1,500 (left) and 12,000 (right). 


\subsection{Mechanical measurements}

To compare the structural response of the different samples and to investigate damage evolution, tests of the initially unstrained specimens under cyclic loading and unloading with fixed amplitude were performed. The stress-strain response of the pure silicone elastomer and the MRE specimens at a strain rate of $0.02 \mathrm{~s}^{-1}$ are shown in Figure 11 and Figure 12, respectively.

For the pure silicone elastomer (Figure 11), there is a small difference in in response between the first cycle and the following ones with only a very weak hysteresis.

In the case of the MRE samples, the nominal stress-strain response of the first cycle differs significantly from the following cycles. The second cycles also differ from the following ones but less significantly. There is a large stress softening during the first cycle, diminishing with the following cycles and becoming negligible after about 6 cycles. The reduction in the stress is almost comparable for the untreated MRE sample (Figure 12a) and the spray-coated MRE sample (Figure 12b) and largest on the primer stirred MRE sample (Figure 12c). After a pre-conditioning of about 6 loading and unloading cycles the stress-strain responses are essentially repeatable having only weak hysteresis for all MREs.

In all samples residual stresses after unloading to the original elongation resulting from viscoelasticity are evident. They are higher for the MRE specimens compared to the pure silicone specimen.

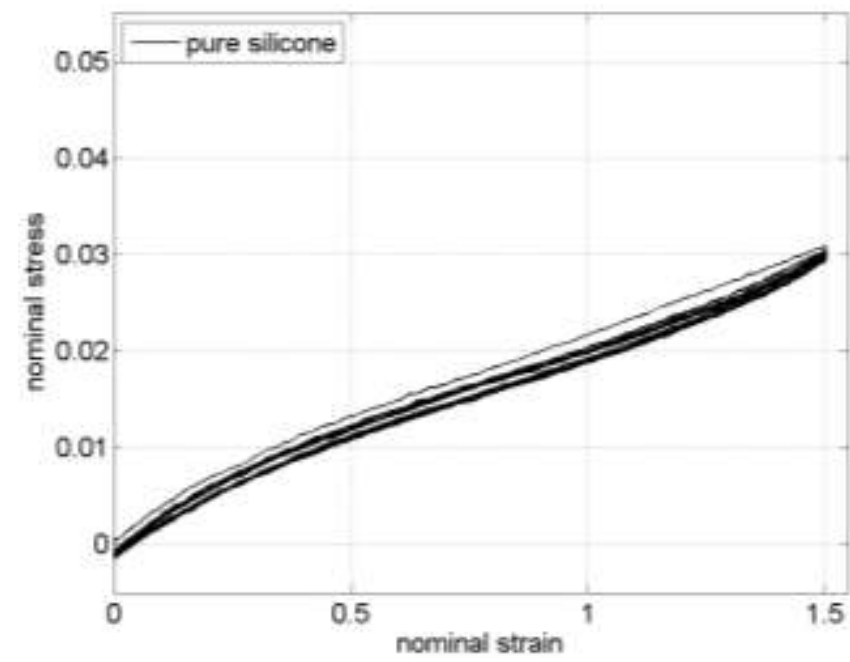

Figure 11: Stress-strain response of the pure silicone elastomer at a strain rate of $0.02 \mathrm{~s}^{-1}$.

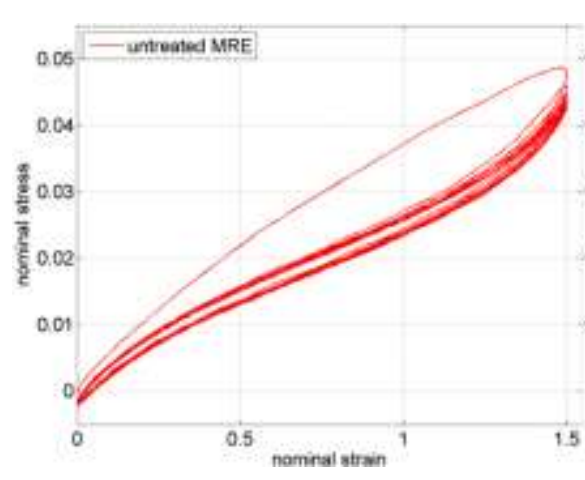

a. untreated MRE

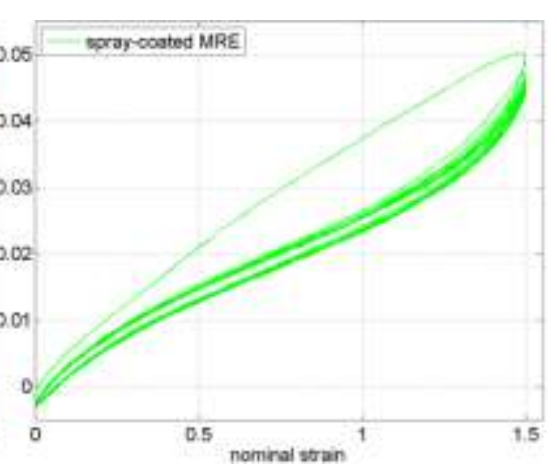

b. spray-coated MRE

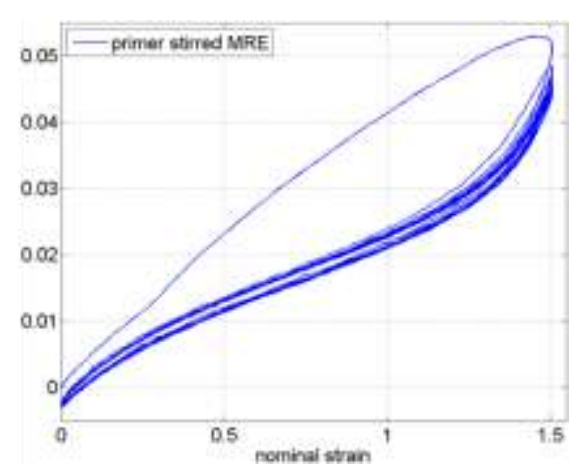

c. primer stirred MRE

Figure 12: Stress-strain responses of the different MRE samples with a particle volume fraction of 3.5\% parallel to particle alignment at a strain rate of $0.02 \mathrm{~s}^{-1}$. 
To better compare the stress-strain response of the different samples at high strain and to confront them to the SEM investigations, the values of nominal stresses corresponding to a strain level of $140 \%$ are reported in Figure 13 . Values for the loading path of the first cycle (dark) and the repeatable sixth cycle (light) point out the damage evolution from the initially unstrained samples.

Compared to the pure silicone elastomer, the MRE samples with a particle volume fraction of 3.5\% show a higher stiffness. The stiffness at the first and sixth cycle of the MREs with untreated and spray-coated particles is almost the same. This confirms the SEM observation that only small amounts of silane primer could be applied to the particles by spraying the primer dilution with an airbrush. Although the stiffness at the first cycle for the primer stirred MRE is increased by the primer treatment, the stiffness at the sixth cycle is lower compared to the other MRE specimens. This goes along with the SEM images showing partial debonding but larger and more numerous crack openings due to large particle agglomerates.

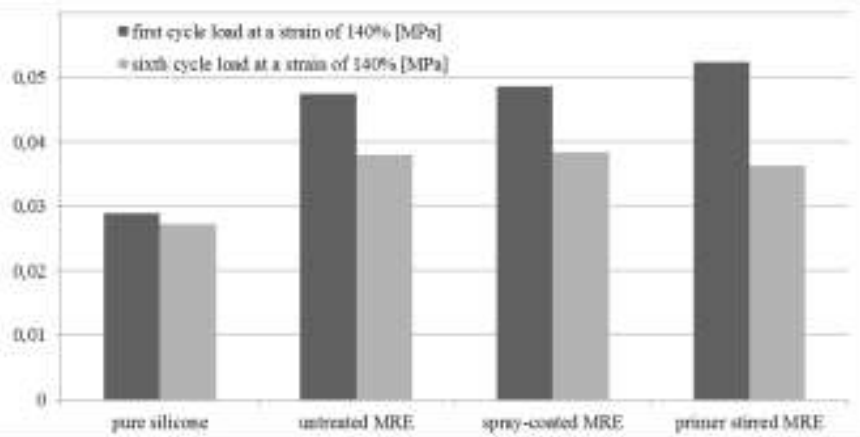

Figure 13: Values of the stresses at the first and sixth cycles of loading corresponding to a nominal strain level of $140 \%$ for all tested samples.

\section{CONCLUSIONS AND PERSPECTIVES}

This work investigates the interfacial adhesion between the iron fillers and the soft silicone matrix in magnetorheological elastomers at high deformations. For this purpose, different MRE samples with 3.5\% particle volume fraction of aligned carbonyl iron powder were prepared modifying the surface of the iron particles by the application of a silane primer, following two different procedures.

The primer spray-coated particles showed just a few well covered particles under SEM. Only a small amount of primer could be applied by this way of treatment. Thus the interfacial adhesion could only be improved at a few locations where we observed well bound rubber at local scale. Therefore just small differences in the microstructure of the aligned chains and in the stress-strain response compared to the MRE sample without primer pretreatment could be observed.

The primer solution stirred particles showed a majority of particles stuck together in large agglomerates that appeared poorly coated. This led to thick particle columns with high spatial distances in the MRE samples, where strong and widespread partial debonding could be observed at high strains even though leaving bound rubber residues. In agreement to these observations, the stress-strain response showed higher stiffness at the first loading path as well as more important dissipative losses in the next cycles, but leading finally to a decreased stiffness at the repeatable sixth cycle.

The primer thus could improve the interfacial adhesion between the iron fillers and the silicone matrix if applied homogeneously on all particles while avoiding aggregates. Consequently, this adds another difficult process step to the fabrication. This process could be ameliorated in the future by spraying a large amount of primer dilution and simultaneously stirring and shearing the particles at high speed. Furthermore a grinding process step during air drying could separate particle agglomerates.

As no debonding was observed for strains up to 50\% under SEM and the primer treatment had no significant reinforcing effects on the repeatable sixth cycle of the nominal stress-strain response, the application of a silane primer with the procedures presented here does not seem necessary. By all means both the pure silicone material and the MRE material have weak hysteresis. Further investigations regarding debonding with higher volume fractions also need to be conducted. 


\section{ACKNOWLEDGEMENTS}

The authors wish to thank Alexandre Tanguy in the name of the whole team of engineers at the solid mechanics laboratory at the Ecole Polytechnique for his contributions to the SEM photos.

\section{REFERENCES}

[1] Carlson, J. D. and Jolly, M. R., "MR fluid, foam and elastomer devices," Mechatronics 10, 555-569 (2000).

[2] Li, W. and Zhang, X., "Research and Applications of MR Elastomers," Recent Patents on Mechanical Engineering 1(3), 161-166 (2008).

[3] Kallio, M., "The elastic and damping properties of magnetorheological elastomers," VTT Publications 565, 6263 (2005).

[4] Velázquez, R., Hernández, H. and Preza, E., "A Portable eBook Reader for the Blind," Proc. IEEE EMBS, 2107-2110 (2010).

[5] Lee, C.H. and Jang, M.G., "Virtual Surface Characteristics of a Tactile Display Using Magneto-Rheological Fluids," Proc. Sensors (Basel) 11(3), 2845-2856 (2011).

[6] Ahmed, S. and Jones, F. R., "A review of particulate reinforcement theories for polymer composites," JMS 25, 4933-4942 (1990).

[7] Leblanc, J. L., "Rubber-filler interactions and rheological properties in filled compounds," Prog. Polym. Sci. 27, 627-687 (2002).

[8] Payne, A. R., "The dynamic properties of carbon black loaded natural rubber vulcanizates II," Journal of App. Poly. Science, 6(21), 368-372 (1962).

[9] Dorfmann, A. and Ogden, R. W., "A constitutive model for the Mullins effect with permanent set in particlereinforced rubber," IJSS 41, 1855-1878 (2004).

[10] Diani, J., Fayolle, B. and Gilormini, P., "A review on the Mullins effect," European Polymer Journal 45, 601$612(2009)$.

[11] Gent, A. N. and Park, B., "Failure processes in elastomers at or near a rigid spherical inclusion," JMS 19, $1947-$ 1956 (1984).

[12] Ramier, J., "Comportement mécanique d'élastomères chargés, Influence de l'adhésion charge-polymère, Influence de la morphologie," PhD INSA (Lyon), 10-47 (2004).

[13] Yatsuyanagi, F., Suzuki, N., Ito, M. and Kaidou, H., "Effects of secondary structure of fillers on the mechanical properties of silica filled rubber systems," Polymer 45, 9523-9529 (2001).

[14]Zhang, X.Z., Gong, X.L., Zhang, P.Q. and Li, W.H., "Existence of Bound-Rubber in Magnetorheological Elastomers and Its Influence on Material Properties," Chin. Journal Chemical Physics 20(2), 173-179 (2007).

[15] Yi, W., Wang, Y., Wang, G. and Tao, X., "Investigation of carbon black/silicone elastomer/dimethylsilicone oil composites for flexible strain sensors," Polymer Testing 31, 677-684 (2012).

[16] Gong, X.L., Zhang, X.Z. and Zhang, P.Q., "Fabrication and characterization of isotropic magnetorheological elastomers," Polymer Testing 24, 669-676 (2005).

[17] Chen, L., Gong, X.L. and Li, W.H., "Damping of Magnetorheological Elastomers," Chin. Journal Chemical Physics 21(6), 581-585 (2008).

[18]Fan, Y.C., Gong, X.L., Xuan, S.H., Zhang, W., Zheng, J. and Jiang, W.Q., "Interfacial friction damping properties in magnetorheological elastomers," Smart Mater. Struct. 20, 035007 (2011).

[19] Wang, Y.L., Hu, Y., Chen, L., Gong, X.L., Jiang, W.Q., Zhang, P.Q. and Chen, Z.Y., "Effects of rubber/magnetic particle interactions on the performance of magnetorheological elastomers," Polymer Testing 25, 262-267 (2006).

[20]Fan, Y.C., Gong, X.L., Jiang, W.Q., Zhang, W., Wei, B. and Li, W.H., "Effect of maleic anhydride on the damping property of magnetorheological elastomers," Smart Mater. Struct. 19, 055015 (2010).

[21]Peignot, P. and Rhodes, K., "Choosing a Silicone Adhesive and Treatment System," Medical Device Technology Materials, 2-4 (2004).

[22] Habenicht, G., [Kleben - Grundlagen, Technologien, Anwendungen], Springer, Berlin \& Heidelberg \& New York, 151-167 (2006). 